\title{
ON BERGMAN'S KERNEL FUNCTION FOR SOME UNIFORMLY ELLIPTIC PARTIAL DIFFERENTIAL EQUATIONS
}

\author{
G. G. WEILL ${ }^{1}$
}

1. We present here a generalization of the theory of Bergman's kernel function for uniformly elliptic partial differential equations of the divergence type

$$
\mathfrak{T} u \equiv \partial / \partial x_{k}\left(a_{i k} \partial u / \partial x_{i}\right)=0 .
$$

It is known that for regular open sets $\Omega$ in $R^{n}$ the expression

$$
M_{\Omega}(u)=\int_{\Omega} a_{i k} \partial u / \partial x_{i} \partial u / \partial x_{k} d X
$$

is a natural norm on the space of regular solutions of $\Re u=0$ vanishing at a point $x_{0} \in \Omega$. It is proved that for $E$ compact in $\Omega, x \in E$

$$
|u(x)|^{2} \leqq K(E) M_{\Omega}(u) \text {. }
$$

The existence of Bergman's kernel $K(x, y)$ and the convergence of its expansion in terms of a complete orthonormal set of functions follows at once. We prove the boundedness of $K(x, y)$ on compact subsets of $\Omega$. A sharp value for $K(E)$ is found to be $\sup _{E} K(x, x)$.

2. We consider partial differential equations of the type

$$
\mathfrak{T} u \equiv \partial / \partial x_{k}\left(a_{i k} \partial u / \partial x_{i}\right)=0, \quad i, k=1, \cdots, n,
$$

where the coefficients $a_{i k} \in C^{(1, \lambda)}$ in a regular region $\Omega \subset R^{n}$. Moreover, $\mathfrak{T}$ satisfies a uniform ellipticity condition

$$
\lambda^{-1} \sum_{i=1}^{n} \xi_{i}^{2} \leqq a_{i k} \xi_{i} \xi_{k} \leqq \lambda \sum_{i=1}^{n} \xi_{i}^{2}
$$

We recall the definition of regularity: let $\Omega$ be a subregion of a region $V \subset R^{n}$. Let $B$ be the open unit ball centered at the origin and let $P$ be the hyperplane $x_{n}=0 . \Omega$ shall be called a regular subregion [1] if:

(I) $\mathrm{Bd} \Omega$ is compact in $V$,

(II) every $x \in \mathrm{Bd} \Omega$ has a neighborhood $N(x)$ and a diffeomorphism $h: N(x) \rightarrow B$ such that $h(N(x) \cap B d \Omega)=B \cap P$ and $h(N(x) \cap \Omega)$ is one

Presented to the Society, April 1965; received by the editors November 16, 1964.

1 This research was supported by the National Science Foundation under Grant No. NSF-GP-184. 
of the two half balls of $B-P$,

(III) $\overline{\mathbf{\Omega}}$ is compact in $V$,

(IV) $\Omega$ and $V-\bar{\Omega}$ have the same boundary in $V$,

(V) each component of $V-\Omega$ is noncompact in $V$.

We assume moreover that $h \in C^{(1, \lambda)}$.

We shall use the following lemmas as applied to regular solutions in $\Omega$ of $\mathfrak{T l} u=0$.

LEMMA I (PoINCARÉ) [2]. If $w, w_{x_{i}}$ are square integrable in a ball $B_{R}$ of radius $R$, and if $\bar{w}$ is the average of $w$ over $B_{R}$, then

$$
\int_{B_{R}}(w-\bar{w})^{2} d X \leqq C\left(B_{R}\right) \int_{B R} \sum_{i=1}^{n}\left(w_{x_{i}}\right)^{2} d X,
$$

where $C\left(B_{R}\right)$ denotes a constant which depends only on $B_{R}$.

Lemma II (J. Moser) [3]. If $u$ is a solution of $\mathfrak{T} u=0$ which is defined in $|x|<2 R$ then, for $|x| \leqq R$

$$
u^{2}(x) \leqq C R^{-n} \int_{|x|<2 R} u^{2} d X,
$$

where $C$ denotes a constant.

We now give a bound for the first derivatives of a regular solution of $\mathfrak{T} u=0$ in terms of

$$
M_{\Omega}(u)=\int_{\Omega} a_{i k} \partial u / \partial x_{i} \partial u / \partial x_{k} d X .
$$

THEOREM I. Let $E$ be a compact subset of $\Omega$. Then for $x \in E$, $u$ a regular solution of $\mathfrak{T l} u=0$, one has

$$
\left|\partial u / \partial x_{k}\right|_{E} \leqq C(E) M_{\Omega}^{1 / 2}(u),
$$

where $C(E)$ denotes a constant depending only on $E$.

Proof. Let $\delta>0$ be defined such that the distance from $E$ to $B d \Omega$ is greater than $4 \delta$. If we denote by $B(x ; R)$ the ball of center $x$ and radius $R$,

$$
B(x, 4 \delta) \subset \Omega \quad \forall x \in E .
$$

Let $x_{0}$ be a point of $E$, and let $G(x ; y)$ be Green's function for $B\left(x_{0}, 2 \delta\right)$. Then:

$$
u(x)=\int_{\operatorname{Bd} B\left(x_{0}, 2 \delta\right)} u(y) \partial / \partial \nu_{y}^{*} G(y ; x) d_{y} \sigma, \quad x \in B\left(x_{0}, 2 \delta\right),
$$


where $\partial / \partial \nu^{*}$ denotes the conormal derivative. Hence (cf. [4])

$$
\partial u / \partial x_{k}=\int_{\mathrm{Bd} B\left(x_{0}, 2 \delta\right)} u(y) \partial / \partial x_{k} \partial / \partial \nu_{\nu}^{*} G(y ; x) d_{y} \sigma, \quad x \in B\left(x_{0}, 2 \delta\right) .
$$

Let

$$
\tilde{u}\left(x_{0} ; 2 \delta\right)=\int_{B\left(x_{0}, 2 \delta\right)} u(x) d x / \int_{B\left(x_{0}, 28\right)} d X
$$

then

$$
\begin{aligned}
& \partial u / \partial x_{k}=\int_{\operatorname{Bd} B\left(x_{0}, 2 \delta\right)}\left(u(y)-\bar{u}\left(x_{0} ; 2 \delta\right)\right) \partial / \partial x_{k} \partial / \partial \nu_{y}^{*} G(y ; x) d_{y} \sigma, x \in B(x, 2 \delta), \\
&\left|\partial u / \partial x_{k}\right| \leqq C \max _{\operatorname{Bd} B\left(x_{0}, 2 \delta\right)}\left|u(y)-\bar{u}\left(x_{0} ; 2 \delta\right)\right| \int_{\text {Bd } B\left(x_{0}, 2 \delta\right)} d_{y} \sigma /|x-y|^{n-1}, \\
& x \in B\left(x_{0}, 2 \delta\right) .
\end{aligned}
$$

Let $\omega_{n}$ be the area of the $n-1$ sphere:

$$
\begin{aligned}
& \left|\partial u / \partial x_{k}\right| \leqq C \omega_{n} \cdot 2^{n-1} \max _{\text {Bd } B\left(x_{0}, 2 \delta\right)}\left|u(x)-\bar{u}\left(x_{0} ; 2 \delta\right)\right|, \quad x \in B\left(x_{0}, \delta\right), \\
& \left|\partial u / \partial x_{k}\right| \leqq C^{\mathbf{I}} \max _{y \in B\left(x_{0}, 2 \delta\right)}\left|u(y)-\bar{u}\left(x_{0} ; 2 \delta\right)\right|, \quad x \in B\left(x_{0}, \delta\right),
\end{aligned}
$$

by the maximum principle. By Lemma II

$$
\left|\partial x / \partial x_{k}\right|^{2} \leqq C^{I I} \int_{B\left(x_{0}, 2 \delta\right)}\left(u(x)-\bar{u}\left(x_{0}, 2 \delta\right)\right)^{2} d X \quad x \in B\left(x_{0}, \delta\right),
$$

and by Lemma I

$$
\begin{aligned}
\left|\partial u / \partial x_{k}\right|^{2} & \leqq C^{\mathrm{III}} \int_{B\left(x_{0}, 48\right)} \sum_{i=1}^{n}\left(\partial u / \partial x_{i}\right)^{2} d X \\
& \leqq C^{\mathrm{III}} \lambda \int_{B\left(x_{0}, 48\right)} \lambda^{-1} \sum_{i=1}^{n}\left(\partial u / \partial x_{i}\right)^{2} d X \\
& \leqq C^{\mathrm{IV}} M_{B\left(x_{0}, 48\right)}(u),
\end{aligned}
$$

and $C^{\mathrm{IV}}$ depends only on $\mathfrak{T}$ and $\delta$. Cover now $E$ by a finite number, say $N$, of balls $B\left(x_{j} ; \delta\right), j=1, \cdots, n$.

Then

$$
\left|\partial u / \partial x_{k}\right|^{2} \leqq C^{v} M_{\Omega}(u), \quad x \in E,
$$

where $C^{\mathrm{v}}=\max _{j} C^{\mathrm{Tv}}$. 
3. Let $\Omega$ be regular, and let $x_{0}$ be fixed in $\Omega$. Consider a compact set $E \subset \Omega$. Let $4 \delta$ be a positive number smaller than the distance from $E \cup\left\{x_{0}\right\}$ to Bd $\Omega$. Cover $E \cup\left\{x_{0}\right\}$ by a finite number of open balls of radius $\delta, B\left(x_{0}, \delta\right), \cdots, B\left(x_{N}, \delta\right)$. A point in each $B\left(x_{i} ; \delta\right)$ can be joined to $x_{0}$ by an arc $\gamma_{i}$ in $\Omega$. Let $4 \delta^{\prime}$ be a positive number smaller than the distance from $\cup \bar{B}\left(x_{i} ; \delta\right) \cup \cup \gamma_{i}$ to $\operatorname{Bd} \Omega$ and cover each $\gamma_{i}$ by a finite number of open balls of radius $\delta^{\prime}$, say $B\left(y_{1}, \delta^{\prime}\right), \cdots, B\left(y_{s}, \delta^{\prime}\right)$.

COROLlARY. Let $u$ be a regular solution of $\mathfrak{T} u=0$ vanishing at $x=x_{0}$, then for $x \in E$

$$
|u(x)|^{2} \leqq K(E) M_{\Omega}(u)
$$

where $K(E)$ depends only on $E$ (and on $x_{0}$ ).

Proof. It follows from the theorem that if $B\left(\bar{x}, 4 \delta^{\prime \prime}\right) \subset \Omega$ where $\operatorname{dist}(\bar{x}, \mathrm{Bd} \Omega)>4 \delta^{\prime \prime}$ then for $x$ such that $|x-\bar{x}|<\delta$

$$
|\operatorname{grad} u|^{2} \leqq C\left(\bar{x}, \delta^{\prime \prime}\right) M_{B\left(\bar{x}, 4 \delta^{\prime \prime}\right)}(u) \text {. }
$$

Let $x^{\prime}, x^{\prime \prime}$ be points in $B\left(\bar{x}, \delta^{\prime \prime}\right)$ then

$$
\left|u\left(x^{\prime}\right)-u\left(x^{\prime \prime}\right)\right| \leqq \int_{x^{\prime}}^{x^{\prime \prime}}|\operatorname{grad} u| d s \leqq 2 \delta^{\prime \prime} C^{1 / 2}\left(\bar{x}, \delta^{\prime \prime}\right) M_{B\left(\bar{x}, 4 \delta^{\prime \prime}\right)}^{1 / 2}(u) .
$$

Applying the last inequality to the covering defined by $B\left(x_{i}, 4 \delta\right)$ and $B\left(y_{j}, 4 \delta^{\prime}\right)$ one gets

$$
\left|u(x)-u\left(x_{0}\right)\right|^{2}=|u(x)|^{2} \leqq K(E) M_{\Omega}(u),
$$

which proves the corollary.

From the corollary and from the general theory [5] we get immediately the existence of a complete orthonormal system $\left\{\phi_{\nu}(x)\right\}$ and an expansion for Bergman's kernel

$$
K(x, y)=\sum_{v=1}^{\infty} \phi_{\nu}(x) \phi_{\nu}(y),
$$

which for fixed $x$ converges uniformly on compact subsets of $\Omega$. The $\phi_{\nu}(x)$ may be chosen so that $\phi_{\nu}\left(x_{0}\right)=0 \forall \nu$.

As an application we shall prove the following theorem.

4. THEOREM II. The function $K(x, x)$ is bounded on every compact subset $E$ of $\Omega$.

Proof. Cf. [6]. From Theorem I we get, for fixed $k$ :

$$
\left|\partial u / \partial x_{k}\right|_{E} \leqq C(E) M_{\Omega}^{1 / 2}(u) .
$$


If $u$ is a solution of $\mathfrak{T} u=0$, regular and such that $\partial u / \partial x_{k}=1$ at $X_{0} \in E$, then $M_{\Omega}(u) \geqq 1 / C^{2}(E)$.

Consider the function

$$
\begin{aligned}
\phi^{*}(x) & =\lambda^{-1 / 2} \sum_{v=1}^{N} \partial \phi_{v} / \partial x_{k}\left(x_{0}\right) \phi_{\nu}(x) / \sum_{v=1}^{N}\left[\partial \phi_{\nu}\left(x_{0}\right) / \partial x_{k}\right]^{2}, \\
\mathscr{T} \phi^{*} & =0 \text { and } \partial \phi^{*} / \partial x_{k}\left(x_{0}\right)=1
\end{aligned}
$$

Therefore

$$
M_{\Omega}\left(\phi^{*}\right) \leqq \lambda \int_{\Omega}\left|\operatorname{grad} \phi^{*}\right|^{2} d X=1 / \sum_{\nu=1}^{N}\left[\partial \phi_{\nu}\left(x_{0}\right) / \partial x_{k}\right]^{2} .
$$

Therefore

$$
\sum_{\nu=1}^{N}\left[\partial \phi_{\nu} / \partial x_{k}\left(x_{0}\right)\right]^{2} \leqq C^{2}(E)
$$

and

$$
\sum_{n=1}^{\infty}\left[\partial \phi_{v} / \partial x_{k}\left(x_{0}\right)\right]^{2} \leqq C^{2}(E)
$$

and this is true for all $x_{0} \in E$. An analogous proof works for all $k$, $k=1, \cdots, n$.

Now, we have

$$
\sum_{v=1}^{N}\left[\phi_{\nu}(x)\right]^{2}=\sum_{v=1}^{N}\left[\phi_{\nu}(x)-\phi_{\nu}\left(x_{0}\right)\right]^{2}
$$

and

$$
\begin{aligned}
{\left[\phi_{\nu}(x)-\phi_{\nu}\left(x_{0}\right)\right]^{2} } & \leqq\left[\int_{\gamma\left(x_{0}, x\right)}\left|\operatorname{grad} \phi_{\nu}\right| d s\right]^{2} \\
& \leqq 4 L^{2} \int_{\gamma\left(x_{0}, x\right)}\left|\operatorname{grad} \phi_{\nu}\right|^{2} d s
\end{aligned}
$$

where $\gamma\left(x_{0}, x\right)$ is an arc from $x_{0}$ to $x$, lying in $\Omega$ and of length $L$; therefore

$$
\sum_{n=1}^{N}\left[\phi_{\nu}(x)\right]^{2} \leqq 4 L^{2} C^{2}(E)
$$

and 


$$
\sum_{r=1}^{\infty}\left[\phi_{p}(x)\right]^{2}=K(x, x) \leqq 4 L^{2} C^{2}(E) .
$$

We are now ready to give the best estimate for $K(E)$ in the corollary. Let $p_{0}$ and $p_{1}$ be the principal functions for $\Re(u=0$ and $\Omega$, defined as in [1]. From Theorem 6 in [1],

$$
|u(x)|^{2} \leqq M_{\Omega}\left(p_{0}-p_{1}\right) M_{\Omega}(u)
$$

with equality only for $u=a\left(p_{0}-p_{1}\right), a \in R$. Moreover, Theorem 5 in [1] shows that

$$
M_{\Omega}(u)-2 u(x)=M_{\Omega}\left(p_{0}-p_{1}\right)+M\left(u-p_{0}+p_{1}\right),
$$

or

$$
u(x)=M_{\Omega}\left(u, p_{0}-p_{1}\right) .
$$

It follows that $p_{0}-p_{1}$, which vanishes at $x=x_{0}$ is the Bergman kernel for the space of regular solutions of $\mathfrak{T} u=0$ in $\Omega$ vanishing at $x_{0}$,

$$
|u(x)|^{2} \leqq K(x, x) M_{\Omega}(u),
$$

and $\sup _{E} K(x, x)$ is the best possible value for $K(E)$.

Another application of the previous results would be the obtention of the extremal properties of principal functions [1] for open regions $V$ of $R^{n}$, such that there exists a nested sequence of regular $\left\{\Omega_{n}\right\}$, with the properties $\Omega_{n+1} \supset \bar{\Omega}_{n}$ and $U_{n} \Omega_{n}=V$.

\section{Bibliography}

1. L. Sario and G. G. Weill, Normal linear operators and second order elliptic partial differential equations, Trans. Amer. Math. Soc. 120 (1965), 225-235.

2. H. Poincare, Sur les equations de la physique mathematique, Rend. Circ. Mat. Palermo 8 (1894), 57-155.

3. J. Moser, A new proof of de Giorgi's theorem concerning the regularity problem for elliptic differential equations, Comm. Pure Appl. Math. 13 (1960), 457-468.

4. G. Fichera, Analisi esistenziale per le soluzioni dei problemi al contorno misti, relativi all 'equazione e ai sistemi di equazioni del secondo ordine di tipo ellittico autoaggiunti, Ann. Scuola Norm. Sup. Pisa (3) 1 (1947), 75-100.

5. —_, Sulla "Kernel functions," Boll. Un. Mat. Ital. 7 (1952), 4-15.

6. S. Bergman, Functions satisfying certain partial differential equations of elliptic type and their representation, Duke Math. J. 14 (1947), 349-366.

YESHIVA UNIVERSITY 